\title{
A NOTE ON HENRICI'S TRIPLE PRODUCT THEOREM
}

\author{
PER W. KARLSSON AND H. M. SRIVASTAVA
}

(Communicated by Andrew Odlyzko)

\begin{abstract}
Making use of certain known transformations in the theory of hypergeometric functions, the authors prove a general triple series identity which readily yields Henrici's recent result expressing the product of three hypergeometric ${ }_{0} F_{1}$ functions in terms of a hypergeometric ${ }_{2} F_{7}$ function.
\end{abstract}

Recently, Henrici [2] derived the elegant formula:

$$
\begin{aligned}
& \text { (1) }{ }_{0} F_{1}\left[\begin{array}{cc}
-; & x \\
6 c ; &
\end{array}\right]{ }_{0} F_{1}\left[\begin{array}{ll}
-; & \omega x \\
6 c ; & \omega
\end{array}\right]{ }_{0} F_{1}\left[\begin{array}{cc}
-; & \omega^{2} x \\
6 c ; &
\end{array}\right]
\end{aligned}
$$

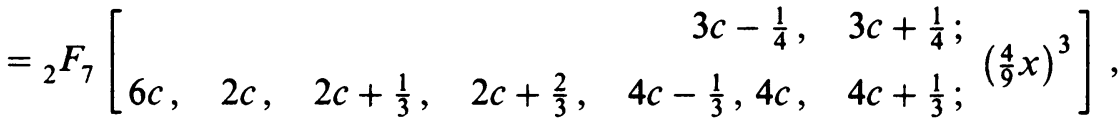

where

$$
\omega=\exp \left(\frac{2 \pi i}{3}\right),
$$

by consideration of the differential equations satisfied by the functions on either side of (1). In the present note we give a shorter proof of (1), utilizing certain known transformations of hypergeometric functions. Indeed, we shall first prove the following general triple series identity:

$$
\begin{aligned}
& \sum_{m, n, p=0}^{\infty} \Delta_{m+n+p} \frac{\omega^{n+2 p}}{(b)_{m}(b)_{n}(b)_{p}} \frac{x^{m+n+p}}{m ! n ! p !} \\
& =\sum_{r=0}^{\infty} \frac{\Delta_{3 r}\left(\frac{1}{2} b-\frac{1}{4}\right)_{r}\left(\frac{1}{2} b+\frac{1}{4}\right)_{r}}{(b)_{r}\left(\frac{1}{3} b\right)_{r}\left(\frac{1}{3} b+\frac{1}{3}\right)_{r}\left(\frac{1}{3} b+\frac{2}{3}\right)_{r}\left(\frac{2}{3} b-\frac{1}{3}\right)_{r}\left(\frac{2}{3} b\right)_{r}\left(\frac{2}{3} b+\frac{1}{3}\right)_{r}} \frac{\left(\frac{4}{9} x\right)^{3 r}}{r !},
\end{aligned}
$$

where $\left\{\Delta_{n}\right\}_{n=0}^{\infty}$ is a bounded sequence of complex numbers, and $\omega$ is defined by (2). For $b=6 c$ and $\Delta_{n}=1 \quad(n \geq 0)$, the identity (3) evidently yields Henrici's formula (1).

Received by the editors March 10, 1989.

1980 Mathematics Subject Classification (1985 Revision). Primary 33A30.

Key words and phrases. Hypergeometric functions, Chu-Vandermonde theorem, Appell functions, triple series identity, quadratic and cubic transformations, Pochhammer symbol. 
Let $\Omega$ denote the left-hand side of (3). By employing some elementary series manipulations, it is not difficult to show that

$$
\Omega=\sum_{k=0}^{\infty} \frac{\Delta_{k} \Lambda_{k}}{(b)_{k}} \frac{x^{k}}{k !}
$$

where

$$
\Lambda_{k}=F_{4}\left[-k, 1-b-k ; b, b ; \omega, \omega^{2}\right]
$$

in terms of the Appell $F_{4}$ function defined by

$$
\begin{gathered}
F_{4}[\alpha, \beta ; \gamma, \delta ; x, y]=\sum_{m, n=0}^{\infty} \frac{(\alpha)_{m+n}(\beta)_{m+n}}{(\gamma)_{m}(\delta)_{n}} \frac{x^{m}}{m !} \frac{y^{n}}{n !}, \\
(\sqrt{|x|}+\sqrt{|y|}<1) .
\end{gathered}
$$

With the aid of the well-known quadratic transformation (cf., e.g., [3, p. 303, Equation 9.4(97)]):

$$
\begin{aligned}
F_{4}[\alpha, \alpha+ & \left.\frac{1}{2}-\beta ; \gamma, \beta+\frac{1}{2} ; x, y^{2}\right] \\
& =(1+y)^{-2 \alpha} F_{2}\left[\alpha, \alpha+\frac{1}{2}-\beta, \beta ; \gamma, 2 \beta ; \frac{x}{(1+y)^{2}}, \frac{4 y}{(1+y)^{2}}\right],
\end{aligned}
$$

we find from (5) that

$$
\Lambda_{k}=\omega^{k} F_{2}\left[-k, 1-b-k, b-\frac{1}{2} ; b, 2 b-1 ; 1,4\right],
$$

since $1+\omega+\omega^{2}=0$ and $\omega^{3}=1$.

Now write this Appell $F_{2}$ function as a single series whose terms involve a terminating ${ }_{2} F_{1}[1]$. Applying the Chu-Vandermonde theorem in order to sum this ${ }_{2} F_{1}[1]$ series, we have

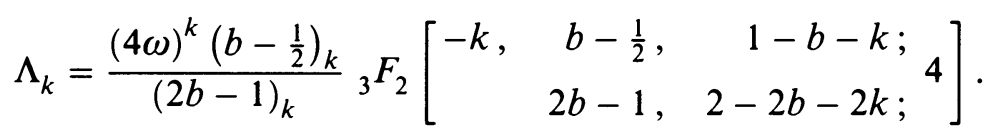

For the polynomial on the right-hand side of $(8)$, we shall now prove the summation formula:

(9)

$$
{ }_{3} F_{2}\left[\begin{array}{ccc}
-k, & b-\frac{1}{2}, & 1-b-k ; \\
2 b-1, & 2-2 b-2 k ;
\end{array}\right]= \begin{cases}0, & k \neq 0(\bmod 3), \\
\frac{2^{-2 r}(3 r) !\left(b-\frac{1}{2}\right)_{2 r}}{r !(b)_{r}\left(b-\frac{1}{2}\right)_{3 r}}, & k=3 r\left(r \in \mathbb{N}_{0}\right),\end{cases}
$$

which, when applied to (8), yields

$$
\Lambda_{3 r}=\frac{2^{4 r}(3 r) !\left(b-\frac{1}{2}\right)_{2 r}}{r !(b)_{r}(2 b-1)_{3 r}}, \quad \Lambda_{3 r+1}=0, \quad \text { and } \quad \Lambda_{3 r+2}=0
$$


Upon substitution from (10) into (5), we observe that

$$
\Omega=\sum_{r=0}^{\infty} A_{r} \Delta_{3 r} x^{3 r},
$$

where

$$
\begin{aligned}
A_{r} & =\frac{\Lambda_{3 r}}{(3 r) !(b)_{3 r}}=\frac{2^{4 r}\left(b-\frac{1}{2}\right)_{2 r}}{(b)_{r}(b)_{3 r}(2 b-1)_{3 r} r !} \\
& =\frac{2^{6 r}\left(\frac{1}{2} b-\frac{1}{4}\right)_{r}\left(\frac{1}{2} b+\frac{1}{4}\right)_{r}}{3^{6 r}(b)_{r}\left(\frac{1}{3} b\right)_{r}\left(\frac{1}{3} b+\frac{1}{3}\right)_{r}\left(\frac{1}{3} b+\frac{2}{3}\right)_{r}\left(\frac{2}{3} b-\frac{1}{3}\right)_{r}\left(\frac{2}{3} b\right)_{r}\left(\frac{2}{3} b+\frac{1}{3}\right)_{r} r !} .
\end{aligned}
$$

This evidently completes the proof of the general triple series identity (3).

In order to prove the summation formula (9), we recall Bailey's cubic transformation [1, p. 249, Equation (4.06)]:

$$
\begin{aligned}
&{ }_{3} F_{2} {\left[\begin{array}{rr}
3 \alpha, \beta, 3 \alpha-\beta+\frac{1}{2} ; & 12 \\
2 \beta, 6 \alpha-2 \beta+1 ; & 4 x
\end{array}\right] } \\
& \quad=(1-x)^{-3 \alpha}{ }_{3} F_{2}\left[\begin{array}{r}
\alpha, \alpha+\frac{1}{3}, \alpha+\frac{2}{3} ; \\
\beta+\frac{1}{2}, 3 \alpha-\beta+1 ;
\end{array} \frac{27 x^{2}}{4(1-x)^{3}}\right]
\end{aligned}
$$

with $3 \alpha$ equal to zero or a negative integer. The apparent singularity at $x=1$ is dealt with by series reversal. First, let $\alpha=-r$, where $r \in \mathbb{N}_{0}$. The right-hand side of (12) may then be transformed as follows:

$$
\begin{aligned}
& (1-x)_{3}^{3 r} F_{2}\left[\begin{array}{c}
-r,-r+\frac{1}{3},-r+\frac{2}{3} ; \frac{27 x^{2}}{4(1-x)^{3}} \\
\beta+\frac{1}{2}, 1-3 r-\beta ;
\end{array}\right] \\
& =\frac{(1-x)^{3 r}\left(\frac{1}{3}-r\right)_{r}\left(\frac{2}{3}-r\right)_{r}}{\left(\beta+\frac{1}{2}\right)_{r}(1-\beta-3 r)_{r}}\left[\frac{-27 x^{2}}{4(1-x)^{3}}\right]^{r} \\
& \times{ }_{3} F_{2}\left[\begin{array}{r}
-r, \frac{1}{2}-r-\beta, 2 r+\beta ; \\
\frac{1}{3}, \frac{2}{3} ;
\end{array} \frac{4(1-x)^{3}}{27 x^{2}}\right] \\
& =\frac{\left(\frac{1}{4} x^{2}\right)^{r}(3 r) !(\beta)_{2 r}}{r !\left(\beta+\frac{1}{2}\right)_{r}(\beta)_{3 r}}{ }_{3} F_{2}\left[\begin{array}{r}
-r, \frac{1}{2}-r-\beta, 2 r+\beta ; \\
\frac{1}{3}, \frac{2}{3} ;
\end{array} \frac{4(1-x)^{3}}{27 x^{2}}\right] .
\end{aligned}
$$

The last expression in (13) is based upon some elementary Pochhammer symbol manipulations. Clearly, we may now take $x=1$, and this proves (9) in the case $k=3 r$. We next perform similar transformations of the right-hand side of (12) in the cases when $\alpha=-r-\frac{1}{3}$ and $\alpha=-r-\frac{2}{3}$, and we find that the exponents of $(1-x)$ do not cancel: we are left with factors $(1-x)$ and $(1-x)^{2}$, respectively. Thus, taking $x=1$, we obtain zero in these cases, and (9) is completely established. 


\section{REFERENCES}

1. W. N. Bailey, Products of generalized hypergeometric series, Proc. London Math. Soc. 28 (1928), 242-254.

2. P. Henrici, A triple product theorem for hypergeometric series, SIAM J. Math. Anal. 18 (1987), 1513-1518.

3. H. M. Srivastava and P. W. Karlsson, Multiple Gaussian hypergeometric series, Halsted Press (Ellis Horwood Limited, Chichester); Wiley, New York, Chichester, Brisbane, and Toronto, 1985.

Physics Laboratory II, Technical University of Denmark, DK-2800 Lyngby, Denmark

Department of Mathematics, University of Victoria, Victoria, British Columbia V8W 2Y2, CANADA 